\section{Secondary prevention of meningococcal disease}

\section{Penicillin is not recommended in British guidelines}

EDrToR,-Bjørn-Erik Kristiansen and ArneBirger Knapskog call for a change in the current British guidelines on the prevention of meningococcal disease without adequately substantiating their arguments. ${ }^{1}$ Their view contradicts the latest guidelines from the Public Health Laboratory Service's Meningococcal Infections Working Group $^{2}$ and will cause confusion when clarity is paramount. The failures in administration of prophylaxis cannot be overcome without clear guidelines and may result in preventable life threatening illness.

The prevention of secondary cases of meningococcal disease in Britain is by chemoprophylaxis with rifampicin, ciprofloxacin, or ceftriaxone for all household and kissing contacts of patients with the disease. ${ }^{2}$ Chemoprophylaxis is effective ${ }^{3}$; resistance to rifampicin is rare in Britain; and parents are advised to seek medical attention if a contact develops fever or a rash, even if months have elapsed since the illness. Kristiansen and Knapskog argue that the addition of penicillin could prevent systemic disease but do not provide convincing evidence to support their claim. The Department of Health and the Communicable Disease Surveillance Centre in Britain do not recommend the use of penicillin, and to advocate a change in policy in

\section{Advice to authors}

We receive more letters than we can publish: we can currently accept only about one third. We prefer short letters that relate to articles published within the past four weeks. Letters received after this deadline stand less chance of acceptance. We also publish some "out of the blue" letters, which usually relate to matters of public policy.

When deciding which letters to publish we favour originality, assertions supported by data or by citation, and a clear prose style. Wit, passion, and personal experience also have their place.

Letters should have fewer than 400 words (please give a word count) and no more than five references (including one to the BMJ article to which they relate); references should be in the Vancouver style. We welcome pictures.

Letters should be typed and signed by each author, and each author's current appointment and address should be stated. We encourage you to declare any conflict of interest.

Please enclose a stamped addressed envelope if you would like to know whether your letter has been accepted or rejected.

Letters will be edited and may be shortened. the middle of the seasonal increase in disease could adversely affect efforts to prevent secondary disease.

Penicillin may be used to treat patients with coprimary infection (that is, those with active disease at the time of presentation of the index case), in which rifampicin is probably insufficient. Such cases, however, are uncommon.

Rifampicin alone is adequate to clear carriage of pathogenic strains of Neisseria in both the index case and contacts. However, most secondary cases occur when there has been a failure in the administration of chemoprophylaxis. ${ }^{4}$ The reasons for such failure are multifactorial but include confusion about and failure to implement chemoprophylactic guidelines when an index case presents; problems with compliance, particularly in young children (for whom intramuscular ceftriaxone might be considered); and failure to appreciate the diagnosis in the index case because of negative results of meningococcal culture or of antigen screening.

Today, owing to the reduction in meningitis due to Haemophilus influenzae type $b$ by vaccination, there is more than an even chance that a child with bacterial meningitis in Britain has meningococcal infection. ${ }^{5}$ When bacterial meningitis is suspected or a child presents with fever and a petechial rash, immediate chemoprophylaxis should be offered to all household and kissing contacts of the case. Adhering to current guidelines for prevention can save lives.

ANDREW J POLLARD ANDREW J POLLARD
Action Research training fellow Consultant paediatrician ROBERT BOOY MICHAEL LEVIN Lecturer Professor

Paediatric Infectious Diseases Unit,

Department of Paediatrics,

St Mary's Hospital Medical School London W2 $1 \mathrm{NY}$

1 Kristiansen BE, Knapskog AB. Secondary prevention of meningococcal disease. $B M$ F 1996;312:591-2. (9 March.)

2 Public Health Laboratory Service Meningococcal Infections Working Group and Public Health Medicine Environmental Group. Control of meningococcal disease: guidance for tal Group. Control of meningococcal disease: guidance for Rep CDR Rev 1995;5:R189-95.

3 Report from the Public Health Laboratory Service Communicable Disease Surveillance Centre. BMF 1986;293:1293-4. Cooke RPD, Riordan T, Jones DM, Painter MJ. Secondary cases of meningococcal infection among close family and cases of meningococcal infection among close family and 1989;298:555-8.

5 Booy R, Hodgson S, Carpenter L, Mayon-White RT, Slack MPE, Macfarlane JA, et al. Efficacy of Haemophilus influenzae type b conjugate vaccine PRP-T. Lancet 1994;344:362-6.

\section{Ceftriaxone or ciprofloxacin should be} considered as first line prophylaxis

EDITOR,-We disagree with several points raised by Biørn-Erik Kristiansen and Arne-Birger Knapskog in their editorial on secondary prevention of meningococcal disease. ${ }^{1}$ Rifampicin eradicates carriage but may not prevent systemic disease. Cases have occurred after prophylaxis with rifampicin as the increased risk lasts for several months. ${ }^{2}$ Hoiby et al state that cases have been reported during treatment with penicillin. ${ }^{3}$ In our opinion, concurrent use of rifampicin and phenoxymethylpenicillin is unsatisfactory, and other agents need to be evaluated urgently. Single dose ciprofloxacin has been shown to be effective in eradication. ${ }^{4}$ Single dose ceftriaxone has been shown to be superior to rifampicin ${ }^{5}$ and has the advantage that it can be given to children and pregnant women. Single dose treatment would have the added benefit of ensuring compliance.

We think that it is now time to consider either ceftriaxone or ciprofloxacin as first line prophylaxis for contacts of patients with meningococcal meningitis.

M I MARTIN Senior registrar, microbiolog N A HUTCHINSON Senior registrar, microbiology I J ELTRINGHAM Senior registrar microbiolog P Y C LEE Senior registrar, microbiology K BIRTHISTLE

Department of Microbiology, Senior registrar, virology

St George's Hospital,

London SW 17 OQT

1 Kristiansen B-E, Knapskog A-B. Secondary prevention of meningococcal disease. $B M^{\prime}$ 1996;312:591-2. (9 March.) 2 Foster G, Panigrahi H, Walker M. Failure of chemoprophylaxis to prevent meningococcal disease. $B M$ f 1986;292:886-7.

3 Hoiby EA, Moe RJ, Lystad A, Froholm LO. Phenoxymethylpenicillin treatment of household contacts of meningococcal disease. Antonie van Leeuwenhoek 1986;52:255-7.

4 Gaunt N, Lambert BE. Single dose ciprofloxacin for the eradication of pharyngeal carriage of Neisseria meningitidis. $\mathscr{f}$ Antimicrob Chemother 1988;21:489-96.

5 Schwartz B, Al-Tobaiqi A, Al-Ruwais A, Fontaine RE, A'Ashi J Hightower AW, et al. Comparative efficacy of ceftriaxone and rifampicin in eradicating pharyngeal carriage of group A Neisseria meningitidis. Lancet 1988; 1:1239-42.

\section{No evidence exists to support use of penicillin}

EdrToR,-Given the current high profile of meningococcal disease in Britain it is extremely important that the $B M \mathcal{F}$ should publish an editorial that is firmly evidence based and reflects current guidance available from experts in infectious diseases and communicable disease control. We are thus concerned about Kristiansen and Knapskog's editorial from Norway, which recommends that high risk contacts should be given chemoprophylaxis and preventive treatment with penicillin. ${ }^{1}$ While the use of chemoprophylaxis is not disputed, we take issue with their recommendation that household members below the age of 15 should be given penicillin for seven days to protect them from contracting the disease. This recommendation is based on data from a single study, which was published in 1986 in a journal not accessible to most British readers. ${ }^{2}$

The 1986 citation reports a case series in which it was observed that close contacts aged under 15 of people with meningococcal disease did not develop the disease when given penicillin. This can be criticised on three counts. Firstly, several close contacts developed meningococcal disease shortly after stopping treatment with penicillin, which suggests that penicillin succeeded only in delaying the onset of disease. Secondly, the assertion that penicillin reduces the number of secondary cases relies on the assumption that the findings of a study carried out in Belgium can be directly extrapolated to Norway. To assume that the two groups are directly comparable disregards one of the fundamental rules of epidemiology. Thirdly, a case 
series is not the method of choice: to show an association between use of penicillin and a decreased frequency of meningococcal disease in close contacts a controlled study is required. It should also be noted that a recent monograph on meningococcal disease states that the use of penicillin in Norway to prevent secondary infection "is no longer recommended." 3

In conclusion, there is no evidence to support the use of penicillin, with or without chemoprophylaxis, to reduce the risk of close contacts developing meningococcal disease. We fear that the only effect of this editorial will be to confuse general practitioners and others who may not be well acquainted with the subject. In our opinion the $B M \mathcal{F}$ should instead have given prominence to the recent guidance on meningococcal disease issued by the Public Heath Laboratory Service's Meningococcal Infections Working Group. ${ }^{4}$

TIM STOKES

Registrar in public health medicine RASHMI SHUKLA

Consultant in communicable disease control PHILIP MONK

Consultant in communicable disease control Leicestershire Health,

Leicester LE5 4QF

1 Kristiansen BE, Knapskog AB. Secondary prevention of meningococcal disease. BMf 1996;312:591-2. (9 March.) 2 Hoiby EA, Moe PJ, Lystad A, Feoholm LO, Bovre K Phenoxymethylpenicillin treatment of household contacts of meningococcal disease patients. Antonie van Leeuwenhoek 1986;52:255-7.

3 Begg N. Outbreak management. In: Cartwright K, ed. Meningococcal disease. Chichester: John Wiley, 1995:285-305.

4 Public Health Laboratory Service Meningococcal Infections Working Group and Public Health Medicine EnvironmenWorking Group and Public Health Medicine Environmen-
tal Group. Control of meningococcal disease: guidance for consultants in communicable disease control. Commun Dis Rep CDR Rev 1995;5:R189-95.

\section{Cases are "unavoidably unpredictable"}

EDITOR,-Bjørn-Erik Kristiansen and ArneBirger Knapskog point out that, typically, 3-4\% of contacts of patients with meningococcal disease carry the pathogenic strain of meningococci but fail to draw out the implications of this. ${ }^{1}$ One of the hardest messages to get across to the public is that it is irrational to focus all attention on an isolated case and close contacts of the patient. At the time that a sporadic case presents there will be many other people in the community who are carrying the same pathogenic strain. Contacts of these community carriers (who are not readily identifiable) are also at slightly increased risk. The reason why particular people develop invasive disease is not known, although age, time of the year, genetic susceptibility, and overcrowding are all associated factors. ${ }^{2}$ More recently it has been suggested that viral infection of the respiratory tract may predispose to meningococcal disease. ${ }^{3}$

The situation is thus one of shifting clusters of risk within the community, with the next case being "unavoidably unpredictable."4

PAUL BINGHAM

Consultant in communicable disease control PETER OLD Director of strategy and public health Isle of Wight Health Commission,

Sandy Lane,

Newport,

Isle of Wight PO3 3ED

1 Kristiansen B-E, Knapskog A-B. Secondary prevention of meningococcal disease. BMF 1996;312:591-2. (9 March.)

2 Public Health Laboratory Service Meningococcal Infections

Working Group and Public Health Medicine Environmental Group. Control of meningococcal disease: guidance for consultants in communicable disease control. Commun Dis Rep CDR Rev 1995;5:R189-95.

3 Communicable Disease Surveillance Centre. Invasive meningococcal disease and influenza. Commun Dis Rep CDRWkly 1995;5:255.

4 Gleick J. Chaos: making a new science. London: Abacus, 1993.

\section{British guidelines should have been} considered

EDITOR,-Guidelines on the management and control of meningococcal disease in England and Wales were published last December. ${ }^{1}$ They were developed after extensive consultation between the Public Health Laboratory Service's Meningococcal Infections Working Group and consultants in communicable disease control and represent a consensus based on best available evidence as it pertains to Britain. It would have been helpful if Bjørn-Erik Kristiansen and ArneBirger Knapskog had considered these guidelines before making policy suggestions for Britain on preventing secondary cases of meningococcal disease. ${ }^{2}$

People living in the same household as a patient with meningococcal disease are at increased risk of the disease. It is unclear whether giving antibiotics to these household contacts reduces the risk, although there is evidence of temporary protection. ${ }^{34}$ In a retrospective study of household clusters in England and Wales between 1993 and 1995 no household contacts developed disease within one to seven days of taking rifampicin, although cases occurred subsequently (L Hastings, unpublished data) Rifampicin will not always clear carriage, but Kristiansen and Knapskog do not provide references to support a policy of a combined antibiotic approach, and we understand that this is not the national policy in Norway. The suggestion that preventive treatment should be confined to those aged under 15 would have no logical basis in Britain as age specific attack rates are higher in 15-19 year olds than 6-14 year olds. ${ }^{5}$

Evidence of protection from randomised controlled trials does not exist and would be difficult to obtain, given the few secondary cases and the likely small differences in efficacy between policies. We believe that current recommendations in England and Wales to give rifampicin to household contacts are reasonable in the light of available evidence. The use of sulphonamides as second line drugs is not advocated because one third of strains of meningococci in England and Wales are resistant to sulphonamides and antibiotic sensitivities are usually not known when prophylaxis is given.

In outbreaks the urgency of the situation means that it is inappropriate for consultants in communicable disease control to wait for the results of surveys of swabs before taking action. We agree that increasing use of polymerase chain reaction testing could make this approach more feasible in future.

New conjugated vaccines against disease due to serogroups $\mathrm{A}$ and $\mathrm{C}$ meningococci hold great promise; vaccines against serogroup $C$ are currently undergoing phase II trials in Gloucester and Oxfordshire. Whatever antibiotic policies are followed for the prevention of secondary cases, most (over $95 \%$ ) cases of meningococcal disease are single, sporadic cases. The greater challenge of primary prevention can be met only through the development of effective vaccines.

PHLS Meningococcal Infectious Working Group, M JONES Public Health Laboratory,

Withington Hospital,

Manchester M20 2LR

1 Public Health Laboratory Service Meningococcal Infections Working Group and Public Health Medicine Environmental Group. Control of meningococcal disease: guidance for consultants in communicable disease control. Commun Dis Rep CRD Rev 1995;5:R189-95.

2 Kristiansen B-E, Knapskog A-B. Secondary prevention of meningococcal disease. $B M Y$ 1996;312:591-2. (9 March.)

Cooke RPD, Riordan T, Jones DM, Painter MJ. Secondary cases of meningococcal infection among close family and household contacts in England and Wales, 1984-7. BMf 1989;298:555-8.

4 Stuart JM, Cartwright KAV, Robinson PM, Noah ND. Does eradication of meningococcal carriage in household contacts prevent secondary cases of meningococcal disease? $B M F$ 1989;298:569-70.

Jones DM, Mallard RH. Age incidence of meningococcal infection England and Wales, 1984-1991. F Infect 1993;27:83-8.

\section{Authors' reply}

EDITOR,-In considering the prevention of secondary cases of meningococcal disease we can divide infected people into two groups: those with incubating infections and carriers. As Andrew J Pollard and colleagues state, the most widely used chemoprophylactic agent, rifampicin, ${ }^{12}$ is satisfactory for eradicating carriage. Penicillin is unsatisfactory. Incubating infections require both eradication and treatment. They usually occur in the patient's close family. ${ }^{3}$ As M J Martin and colleagues and Pollard and colleagues agree, rifampicin is not reliable for treating incubating infections. Begg states that penicillin is more reliable than rifampicin for treating incubating infections, ${ }^{1}$ and Wall et al advocate the use of parenteral penicillin to prevent some of the early infections. ${ }^{4}$ We therefore advocate the Norwegian practice of giving penicillin to susceptible household contacts. Treatment with penicillin provides protection from disease as long as it is maintained, but it fails to tackle the problems of (re)infection from adult contacts or the persistence of meningococci in the patient's throat flora. This is perhaps why the practice has been criticised and why we insist that it must be combined with prophylaxis with rifampicin for all close contacts.

Does oral penicillin cure incubating systemic meningococcal disease or does it, as Tim Stokes and colleagues suggest, merely hold the infection in check? We know that oral penicillin protects while it is maintained; more than 4000 households in Norway have been treated in this way without any breakdown of treatment being reported. We also know that parenteral penicillin has been successfully used to treat systemic infections for decades. The fact that oral penicillin cures incubating infection remains unproved, but do we have a better alternative for dealing with the threat of incubating disease in close contacts?

In our opinion the most important way of preventing secondary meningococcal disease, apart from vaccination, is to eradicate carriage by chemoprophylaxis. We believe that giving supplementary oral penicillin to susceptible household members provides added protection. If it could be shown that single dose ceftriaxone or ciprofloxacin cures incubating meningococcal disease as well as eradicating carriage then we would agree with Martin and colleagues that these drugs would be excellent alternatives for preventing meningococcal disease.

It was not the purpose of the editorial to recapitulate the British guidelines; had it been, the editor would surely not have invited Norwegians to write it. Unfortunately, the British guidelines were not available to us when we wrote it last December.

Telemark Biomedical Centre,

BJØRN-ERIK KRISTIANSEN

PO Box 1868 Gulset,

$\mathrm{N}-3705$ Skien,

Norway

N-3700 Skien

ARNE-BIRGER KNAPSKOG County medical officer of health

Begg N. Outbreak management. In: Cartwright K, ed. Menin gococcal disease. Chichester: John Wiley, 1995: 285-305.

2 Harrison LH. The worldwide prevention of meningococcal infection. $\Im A M A$ 1995;273:419-21.

3 Olcén P, Kjellander J, Danielsson, Lindquist BL. Epidemiology of Neisseria meningitidis: prevalence and symptom from the upper respiratory tract in family members to patients with meningococcal disease. Scand 9 Infect Dis 1981;13:105-9.

4 Wall RA, Hassan-King M, Thomas H, Greenwood BM. Meningococcal bacteraemia in febrile contacts of parients with meningococcal disease. Lancet 1986;ii:624. 